\title{
Artrópodos en resina con fines didácticos y de exhibición
}

\author{
Arthropods in resin for didactic and exhibiting aid \\ Rodrigo Javier lópez Plantey', Viviana Noelia Quiroga1, \\ Paola Noemí Turienzo ${ }^{1}$, Miriam Gladys holgado ${ }^{1}$
}

\section{RESUMEN}

Este artículo tiene el propósito de brindar una ayuda didáctica en la enseñanza de la entomología o artrópodos en general, como recurso potencial de enseñanza para las ciencias en el entorno universitario (Agronomía, Veterinaria, Biología, Medicina y otros), así como un recurso válido en otros niveles o talleres de extensión a público en general.

Palabras clave: arañas, insectos, enseñanza, reconocimiento, trabajos prácticos.

\begin{abstract}
The following note is intended to provide a didactic aid in the teaching of entomology or arthropods in general, as a potential teaching resource for the sciences in the university environment (Agronomy, Veterinary, Biology, Medicine and others), as well as a valid resource at other levels or extension workshops to the general public.
\end{abstract}

Keywords: spiders, insects, teaching, recognition, practice work.

\section{Introducción}

La enseñanza de las ciencias requiere de un enfoque holístico y adecuado para brindar una imagen de ciencia realista y racionalista. Para muchos didactas de las ciencias, la educación científica, para ser significativa, debe generar cambios epistémicos, cognitivos, discursivos, conductuales, materiales y sociales en clases (Adúriz- Bravo, 2001).

Desde la entomología, se vienen empleando propuestas didácticas novedosas, tales como la implementación del uso del cómic (Robles-Piñeros, 2013) o de sellos postales (Turienzo, 2018).

Los métodos tradicionales de preparación de artrópodos para su estudio y observación los dejan muy expuestos a roturas (montajes simple, doble, conservación en líquidos), pero se han seguido empleando a través de los años. Sin embargo, algunas prácticas como la inclusión de ejemplares en resina plástica no se utilizan con frecuencia.
El objetivo de esta contribución es realizar una colección de artrópodos en resina con fines educativos y presentarla como alternativa didáctica en su implementación (colaborando con la diversidad, dado que no exigiría la reposición constante del material para las sucesivas utilizaciones en docencia).

\section{Materiales y métodos}

Para la preparación se empleó resina de poliéster no saturada, un catalizador recomendado, un molde adecuado al tamaño del artrópodo a incrustar, guantes descartables, mascarillas descartables, papel de lija "no past" número 120, 180 y 240, papel de lija al agua número 360 y 600, pasta para pulir y telas de pulido.

La técnica consiste en generar en el molde diferentes capas de resina (en un ambiente ventilado y a temperatura de $20-25^{\circ} \mathrm{C}$ ). Una primera base de sostén del artrópodo a incrustar, una segunda que cubra al artrópodo y una tercera de finalización (el

\footnotetext{
1 Facultad de Ciencias Agrarias, Universidad Nacional de Cuyo. Mendoza, Argentina.

* Autor por correspondencia: mholgado@fca.uncu.edu.ar; paolaturienzo@gmail.com
} 
ejemplar debe encontrarse en posición entomológica adecuada). Una vez solidificada (aproximadamente $24 \mathrm{~h}$ ) la resina se desmolda y se pule la superficie a fin de lograr un acabado perfecto que permita observar sin dificultades el ejemplar incrustado. Para su correcta identificación, al momento de incrustar el ejemplar se coloca a escasos centímetros de este una etiqueta con la correspondiente información: nombre científico, nombre vulgar, lugar de recolección, sexo (de ser posible). El tiempo medio de preparación por ejemplar ronda los tres días.

Las ventajas que presenta dicho material didáctico son que permite a estudiantes de todos los niveles manipular tranquilamente los ejemplares, observarlos en $360^{\circ}$, colocarlos bajo microscopio estereoscópico y visualizar detalles. Todo ello sin exponer el material a roturas y al deterioro por insectos que se alimentan de productos de naturaleza proteica animal, entre ellos, colecciones de diversa índole (Rosello, 2001) como las entomológicas (Almeida y Mise, 2009). Esto, a su vez, es fundamental porque no se debería invertir tanto tiempo en actividades de curación o tener un curador a cargo.

Dos ventajas de su implementación son que ayuda al concepto de preservación de la biodiversidad a nivel de especie (Pascual
Trillo, 1999) al no tener que reponer material constantemente y que no lleva preservantes.

Desde un punto de vista didáctico ofrece la posibilidad de analogías con la preservación de artrópodos en ámbar a nivel evolutivo. El ámbar es una gema orgánica de aspecto vítreo y generalmente dorado, que se ha formado a partir de resina de varias especies de árboles prehistóricos, principalmente coníferas (Lanteri et al., 2001). El uso de analogías es un fuerte componente en la enseñanza de las ciencias (Adúriz- Bravo, 2001).

\section{Resultados y proyecciones futuras}

Hasta el momento se cuenta con ejemplares de cinco familias de Arachnidae (Lycosidae, Sparassidae, Theraphosidae, Theridiidae y Sicariidae) y siete órdenes de Insecta (Lepidoptera, Coleoptera, Orthoptera, Hymenoptera, Heteroptera, Diptera y Blattaria). Figura 1.

Dicho material fue recolectado en zonas cultivadas y de piedemonte de la Provincia de Mendoza, especialmente para la realización de esta colección. El material se utiliza actualmente en la asignatura de Zoología Agrícola de la carrera de Ingeniería Agronómica, Departamento de Ciencias Biológicas, de la Facultad de Ciencias Agrarias de la UNCuyo, y en visitas guiadas que se realizan

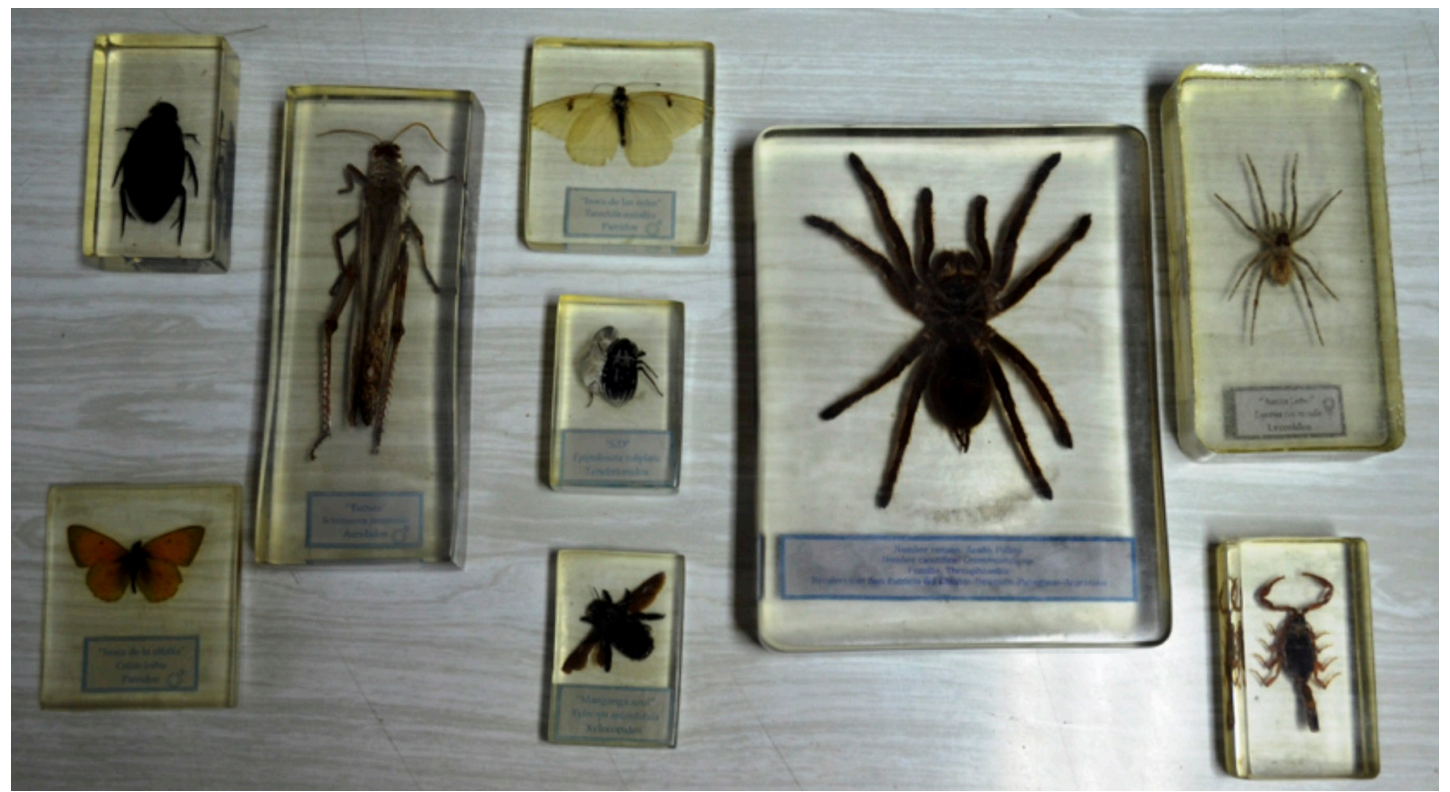

Figura 1. Ejemplares disponibles conservados en resina. 
en la Facultad, donde se recibe a alumnos de nivel inicial y primario dentro de diferentes proyectos de Extensión Universitaria. Esta es una innovación pedagógica, que en un futuro puede servir para el mantenimiento de ejemplares de la colección entomológica que posee dicha cátedra y en proyectos fuera de la universidad, para extensión de temas de interés a público en general, rompiendo así los mitos arraigados en varios sectores de la sociedad (Pérez-Velázquez, 2011).

\section{Literatura Citada}

Adúriz-Bravo, A.

2001. Integración de la epistemología en la formación del profesorado de ciencias. Universitat Autònoma de Barcelona. Departament de Didàctica de les Matematiquesi de les Ciencies Experimentals. Bellaterra. 622 p.

Almeida, L.M.; Mise, K.M.

2009. Diagnosis and key of the main families and species of South American Coleoptera of forensic importance. Revista Brasileira de Entomologia, 53 (2): 227-244.

Lanteri, A.A.; Durante, S.P.; Suárez, S.M.

2001. El ámbar y la historia evolutiva de los insectos. Museo, 3 (15): 15-21.

Pascual Trillo, J.A.

1999. 8 preguntas para una situación desesperada: la conservación de la biodiversidad. $C d G, 7: 38-45$.
Pérez-Velázquez, M.D.

2011. Miedo, pánico y terror a los insectos, reales e imaginarios, entomofobia y otras enfermedades. En: Navarrete-Heredia, J.L.; Castaño Meneses, G.; Quiroz-Rocha, G.A. (Eds.). Facetas de la Ciencia: Ensayos sobre Entomología Cultural. Universidad de Guadalajara. Guadalajara, México. Pp. 49-50.

Robles-Piñeros, J.

2013. Los insectos como estrategias didácticas en la enseñanza de la ecología, a través del cómic. Biografía, 6 (10): 11-21.

Rosello, E.

2001. Plagas de coleópteros en las momias del Museo Arqueológico San Miguel de Azapa. Boletín -e Azeta. Disponible en: www.uta.cl/masma/azeta/dermes. Consultado: 29/9/2018.

Turienzo, P.

2018. La enseñanza de la entomología mediante sellos postales como recurso didáctico. Idesia, 36 (3): 119-129. 
